\title{
A Low Cost SMT Integrated Frequency Doubler and Power Amplifier for 30GHz DBS Uplink Applications
}

\author{
Monica Bhatnagar and Henrik Morkner \\ Agilent Technologies, Inc. 350 W. Trimble Road, San Jose, California, 95131 USA \\ Contact: Henrik Morkner, Phone: (408) 435-6513, FAX: (408) 435-4801 email: henrik_morkner@agilent.com
}

\begin{abstract}
A SMT (Surface Mount Technology) MMIC (Monolithic Microwave Integrated Circuit) frequency multiplier (x2) and power amplifier has been designed for DBS (Direct Broadcast Satellite) and other commercial applications. Using PHEMT technology this MMIC takes a $15 \mathrm{GHz}$ input signal and passes it through a harmonic frequency doubler and then through three stages of power amplification at $30 \mathrm{GHz}$. This MMIC provides an output power of $21 \mathrm{dBm}$, fundamental suppression of $45 \mathrm{dBc}$ and is stable across all voltages and temperatures. This single MMIC SMT solution frequency multiplier and power amplifier is unique compared to any known or published data.
\end{abstract}

\section{Introduction}

One of the problems with DBS (Direct Broadcast Satellite) is returning data from the consumer to the provider. Return information requires only a low data rate and is usually conveyed by modem or voice over land line. Numerous return data items such as pay-per-view, billing, configuration changes, local programming orientation, gaming, etc. could make DBS more effective if transparently done. [6-10]

One solution being implemented in Europe and Asia is to use a $30 \mathrm{GHz}$ uplink frequency for low data rate return. The system uses the same antenna and cable with only additional TV top box and LNB changes. Uplink is carried on the coax just above the receive band $(2.5 \mathrm{GHz})$ then multiplied and amplified for transmit at $30 \mathrm{GHz}$. A simple representation is shown in Figure 1.

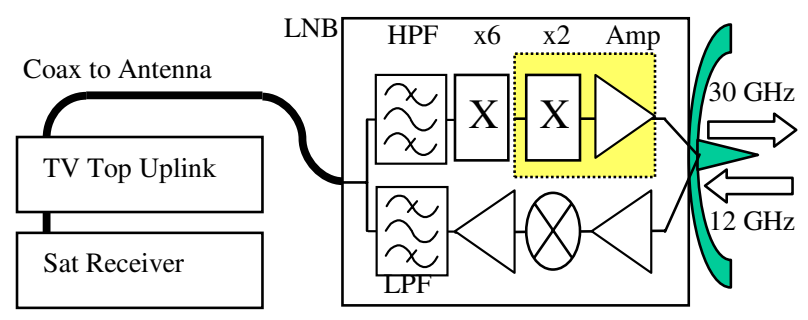

Figure 1. DBS $30 \mathrm{GHz}$ uplink system
This paper focuses on the $15 \mathrm{GHz}$ to $30 \mathrm{GHz}$ frequency multiplier and $100 \mathrm{~mW}$ power amplifier that are integrated together to form the last stage in the uplink. The MMIC had to be SMT (Surface Mount Technology) compatible, low cost, and high performance. The paper being proposed poses the numerous challenges faced and innovative solutions used to develop this high volume $\mathrm{mmW}$ product.

\section{MMIC Process and CAD Tools}

The 6-inch wafer-based mm-wave PHEMT process uses a $0.15 \mu \mathrm{m}$ gate, and has an $f_{T}$ of $85 \mathrm{GHz}$. The wafer material is selected for high power and breakdown with some compromises for noise. A Ti/Pt/Au T-gate is used for low input resistance and high reliability. This PHEMT process is designed to operate with a peak drain voltage up to $5.5 \mathrm{~V}$, and is equipped with passive components which include a $150 \Omega / \square$ bulk resistor, $50 \Omega / \square$ Thin-film resistor, a $0.39 \mathrm{fF} / \mu \mathrm{m}^{2}$ MIM capacitor, a backside via process, and two metal layers for transmission lines. [2, 4-5]

The process includes $100 \%$ on-wafer MMIC test and verification of performance. Passive structures were simulated using Agilent's ADS and Momentum EM software.

\section{Frequency Doubler and Amplifier Design}

A simplified schematic of the frequency doubler and amplifier (QMMP-6130) is shown in Figure 2. The one-stage frequency doubler relies on the non-linear behavior of the FET to produce the doubled signal at the output. A high-pass filter at the input shorts any reflected $2^{\text {nd }}$ harmonic signal to ground. Theoretically, this filter does not affect the $15 \mathrm{GHz}$ excitation signal. The input also consists of matching components tuned for $15 \mathrm{GHz}$. An additional LC-filter is included at the input for stability. The doubler is operated at pinchoff to create a half-wave conduction angle ideal for generation of the $2^{\text {nd }}$ harmonic. When the MMIC is operated at cooler temperatures $(-20 \mathrm{C}$ to $-40 \mathrm{C})$ the $\mathrm{k}$-factor of the doubler degrades twice as fast as an amplifier operated in the linear region. In order to 
ensure stability at all temperatures and biases, the LC-filter is designed to resonate at $30 \mathrm{GHz}$. The filter looks like an open at $30 \mathrm{GHz}$. Since a high reactance is presented to the input of the doubler, $\mathrm{S} 21$ drops at $30 \mathrm{GHz}$. Below $30 \mathrm{GHz}$ the filter looks capacitive, and has little effect on the $15 \mathrm{GHz}$ signal. The shunt inductor at the output short circuits the drain at the fundamental, and passes the $2^{\text {nd }}$ harmonic through. [3]

A medium power amplifier follows the frequency doubler, and includes three cascaded stages designed to deliver maximum power and stable gain at $30 \mathrm{GHz}$. The interstage matches are optimized for narrowband gain and power at $30 \mathrm{GHz}$.

Biased to $\mathrm{Vds}=4 \mathrm{~V}$ the frequency doubler and power amplifier are designed to provide $21 \mathrm{dBm}$ of output power at $30 \mathrm{GHz}$ and 16.5 to $17 \mathrm{~dB}$ of large signal gain. The fundamental suppression of the high and low pass filter network is $45 \mathrm{dBc}$.

The layout is efficient since this is a high volume application where cost is paramount. The prototype layout is shown in Figure 3 with the final production layout approximately $20 \%$ smaller. The layout of the MMIC is designed with yield and reliability in mind including bond pad size, and automated assembly handling.

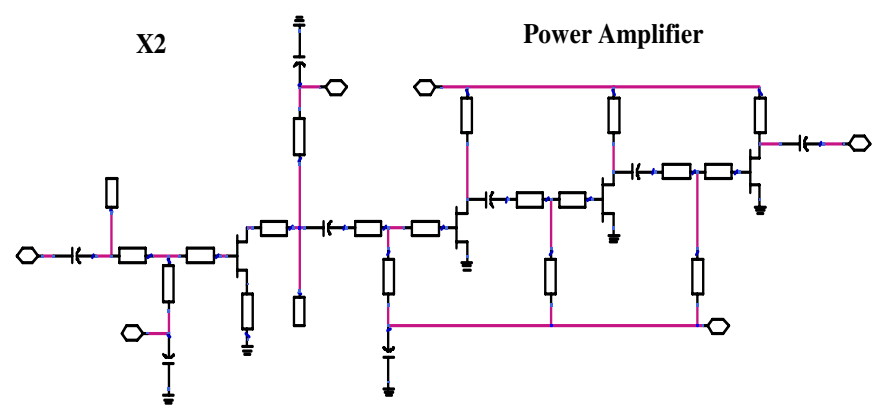

Figure 2. Simplified circuit

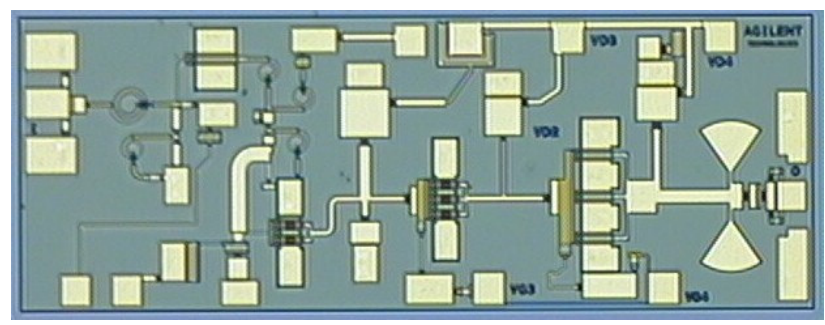

Figure 3. MMIC Photograph

Die size $=1920 \times 760$ micro-meters

\section{Packaging}

The package is $5 \times 5 \mathrm{~mm}$ in dimension and has full surface mount capability. It takes into account the parasitics involved with routing a highfrequency signal up and through the substrate. Built with Rogers 4350 material, the package has a metal backside, making it fully compatible with modern low cost, high volume assembly and test in panel form. [2, 5]

The design of the input and output backside coplanar to topside micro-strip transitions was completed using EEsof's three-dimensional simulation tool, HFSS. With extensive modeling the package I/Os are capable of maintaining better than $15 \mathrm{~dB}$ of return loss up to $40 \mathrm{GHz}$. Compensation for bondwire inductance is included in the package micro-strip launch.

The MMIC is assembled in the package using automated bonders while still in panel form. Lids are then epoxy attached forming an air cavity above the device for maximum performance. Last, the panel is $100 \%$ tested at microwave frequencies to ensure performance for the customer.

Figure 4 shows a 3 dimensional representation and 2 dimensional side view of the package and MMIC. Figure 5 shows the MMIC in the package as assembled. The package design was developed at Agilent for use up to $60 \mathrm{GHz}$ [1].

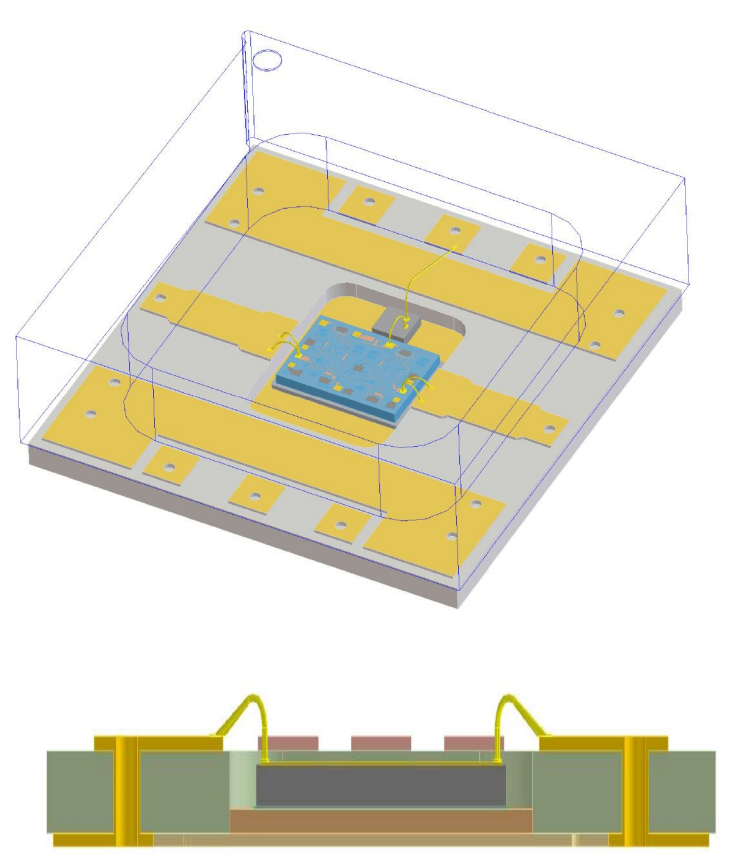

Figure 4. DC-40 GHz low cost package 


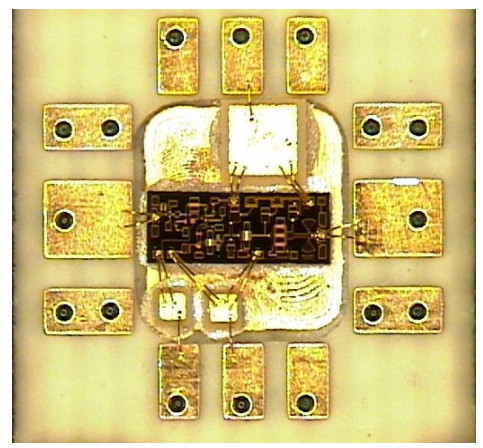

Figure 5. QMMP-6130 as assembled

\section{Measured Results}

Large signal and small signal measurements were taken on the QMMP-6130. A HP8565E spectrum analyzer and HP83650A power source were used to take harmonic measurements and a HP8510C was used to take all small signal measurements. All measurements described in this section were taken at $\mathrm{Vds}=4 \mathrm{~V}, \mathrm{Vg} 1=-1.2 \mathrm{~V}$ and $\mathrm{Vg} 2=-0.5 \mathrm{~V}$. Figure 6 shows the pin configuration for the RF and bias lines. The evaluation board used in these measurements is made out of the Rogers 4350 material, and is shown in Figure 7.

It is important to note that the QMMP-6130 is designed for the input power level to drive the frequency multiplier into saturation. This requires the input power to be contained in a narrow range, for this part 0 to $+5 \mathrm{dBm}$. The resultant output power range is approximately +17 to $21 \mathrm{dBm}$. There is no small signal gain value for this part as it must be in large signal compression (with about 17 $\mathrm{dB}$ of gain) to operate.

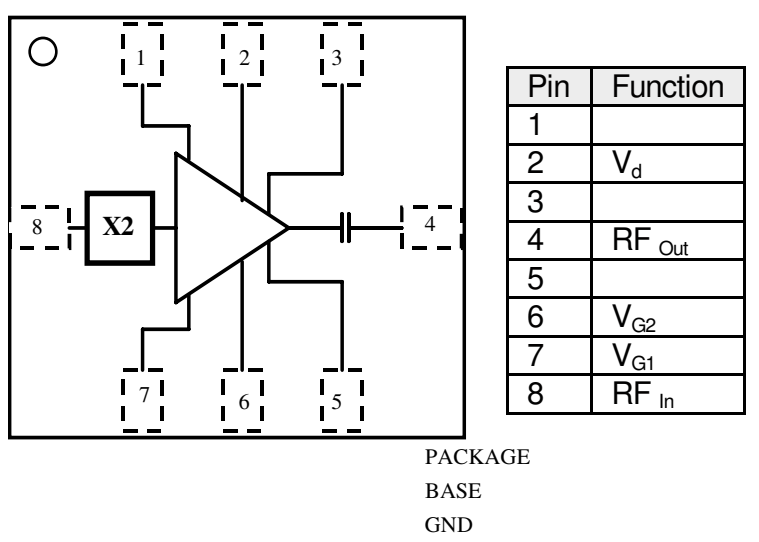

Figure 6. RF and DC pin configuration

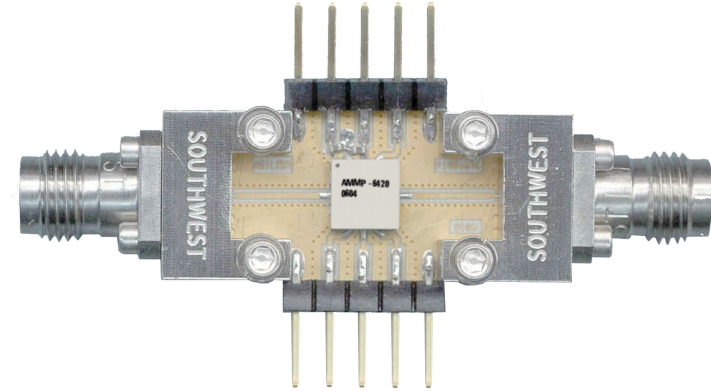

Figure 7. Evaluation module

Figures 8 and 9 describe the large signal response of the QMMP-6130 MMIC. At 5dBm (15GHz) input power, the output power is $21.10 \mathrm{dBm}$ at $30 \mathrm{GHz}$. $2 \mathrm{H}-1 \mathrm{H}$, or the fundamental suppression is approximately $45 \mathrm{dBc}$. Also shown are $1 \mathrm{H}, 2 \mathrm{H}$ and $3 \mathrm{H}$ vs. frequency (see figure 9). The $2^{\text {nd }}$ harmonic signal peaks slightly around $30.5 \mathrm{GHz}$ and the fundamental and $3^{\text {rd }}$ harmonics decrease as frequency increases.

\begin{tabular}{|c|c|c|}
\hline Pin (dBm) & Pout (dBm) & $\begin{array}{c}\text { H1 Suppression } \\
\text { (dBc) }\end{array}$ \\
\hline 0.00 & 16.80 & 44.50 \\
\hline 1.00 & 18.30 & 44.70 \\
\hline 2.00 & 19.30 & 45.60 \\
\hline 3.00 & 20.10 & 46.60 \\
\hline 4.00 & 20.80 & 47.70 \\
\hline 5.00 & 21.10 & 48.70 \\
\hline 6.00 & 21.40 & 49.00 \\
\hline 7.00 & 21.60 & 48.90 \\
\hline
\end{tabular}

Figure 8. Output Power and Fundamental Suppression

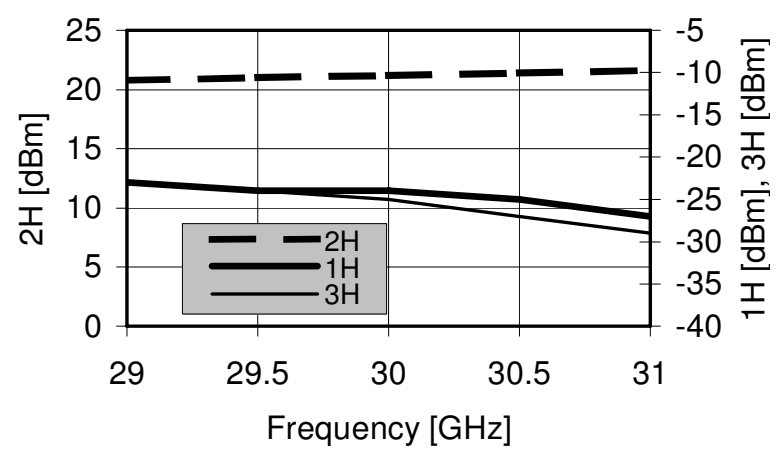

Figure 9. $1 \mathrm{H}, 2 \mathrm{H}$ and $3 \mathrm{H}$ Output Power vs. Output Frequency 
Figure 10 shows the spectrum of the MMIC where the $15 \mathrm{GHz}$ input signal power is $5 \mathrm{dBm}$. The fundamental and $3^{\text {rd }}$ harmonic are far lower than the $2^{\text {nd }}$ harmonic, and there are no spurs or signs of oscillation present. In fact, the spectrum, at $-40 \mathrm{c}$, is clean for a wide range of $\mathrm{Vds}$ values $(1.8$ to $4.5 \mathrm{~V}$ ) at all power levels. Figure 11 shows the input and output returnloss of the MMIC. The output is matched from $20 \mathrm{GHz}$ to $33 \mathrm{GHz}$ and the input is matched close to $15 \mathrm{GHz}$.

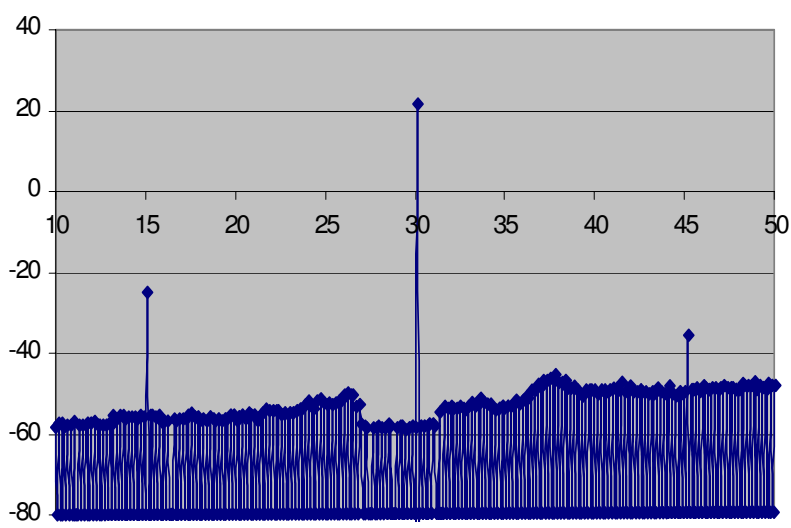

Figure 10. Spectrum Pin=5 dBm at $15 \mathrm{GHz}$

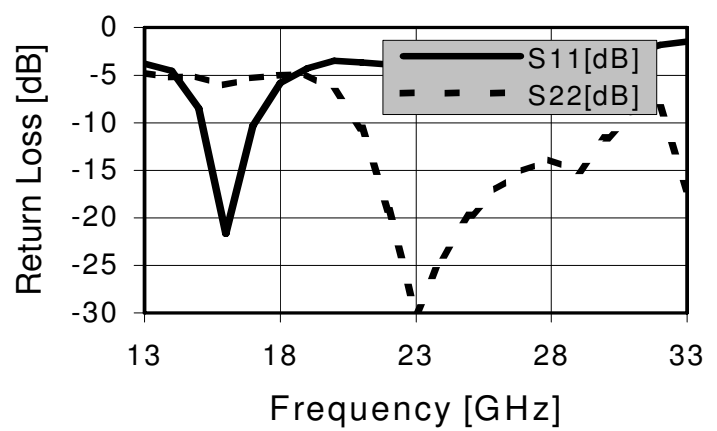

Figure 11. S11 and S22 of the QMMP-6130

\section{Conclusion}

An SMT integrated frequency doubler and power amplifier for $30 \mathrm{GHz}$ commercial communications systems has been designed, fabricated and characterized. With an input power level of $5 \mathrm{dBm}(15 \mathrm{GHz})$, this MMIC provides $21 \mathrm{dBm}$ of output power at $30 \mathrm{GHz}$. The fundamental suppression is $45 \mathrm{dBc}$ and the QMMP6130 is stable across a wide range of $\mathrm{Vds}$ values at all power levels and temperatures down to $-40 \mathrm{c}$. It is designed for low cost utilizing an innovative
SMT package and a volume 6" wafer fabrication facility. The MMIC provides a cost effective alternative to discrete FET designs and chip-andwire hybrid MMICs. This frequency doubler and power amplifier MMIC provides the best combination of cost, performance, and ease of application than any known product advertised or published.

The authors wish to thank all the people that made this product. This includes: Agilent Technologies, San Jose, California, USA: Hue B. Tran, Michael Vice, Sushil Kumar, and Kohei Fujii

\section{References}

[1] D. Nicholson, "Low Return Loss DC to $60 \mathrm{GHz}$ SMT Package with Performance Verification by Precision 50 Ohm Load", 2005 EuMW, Paris, October, 2005

[2] K. Fujii, H. Morkner, "Two Novel Broadband MMIC Amplifiers in SMT Package for 1 to $40 \mathrm{GHz}$ Low Cost Applications", 2005 EuMW, Paris, October, 2005

[3] S.A. Maas, The $R F$ and Microwave Circuit Design Cookbook, Artech House, pp. 125-134, 1998

[4] K. Fujii, H. Morkner, "A 6-30GHz Image-rejection Distributed Resistive MMIC Mixer in a Low Cost Surface Mount Package", 2005 IEEE MTT-s Conference, June 2005

[5] K.Phan, H.Morkner, "A High Performance Yet Easy to Use Low Noise Amplifier in SMT Package for 6 to 20 $\mathrm{GHz}$ Low Cost Applications", 2004 EuMW, Amsterdam, October 2004

[6] Mead, Donald C, Direct Broadcast Satellite Communications, Addison-Wesley/Prentice Hall, NJ, 2000.

[7] Kadish, Jules E, and Thomas WR East, Satellite Communication Fundamentals, Artech House, 2000.

[8] Rappaport, TS, Wireless Communications: Principles \& Practice, Prentice-Hall, NJ, 1996

[9] Benoit, H, Satellite Television, Arnold Publishing, 1999.

[10]Jurgen, Ronald K, Digital Consumer Electronics Handbook, McGraw-Hill, New York, 1997 